\title{
Schizophrenia with the 22q11.2 deletion and additional genetic defects: case history
}

M. Toyosima, * M. Maekawa,* T. Toyota, Y. Iwayama, M. Arai, T. Ichikawa, M. Miyashita,

T. Arinami, M. Itokawa and T. Yoshikawa

\section{Summary}

The 22q11.2 deletion is the most prominent known genetic risk factor for schizophrenia, but its penetrance is at most approximately $50 \%$ suggesting that additional risk factors are required for disease progression. We examined a woman with schizophrenia with this deletion for such risk factors she had high plasma pentosidine levels ('carbonyl stress') and a frameshift mutation in the responsible gene, GLO1. She also had a constant exotropia, so we examined the
PHOX2B gene associated with both schizophrenia and strabismus, and detected a 5-alanine deletion. We propose that the combination of these genetic defects may have exceeded the threshold for the manifestation of schizophrenia.

\section{Declaration of interest}

None.
Schizophrenia is a debilitating and relatively common mental illness, with a prevalence of approximately $1 \%$ worldwide. Although the exact aetiology is unknown, the genetic pathology is thought to consist of numerous weak, risk-conferring genomic variations and/or rare, large-effect variations. An example of the latter is copy number variants (CNVs), typified by the $22 \mathrm{q} 11.2$ deletion syndrome (22q11.2DS; also known as the velocardiofacial syndrome or DiGeorge syndrome $)^{1}$ caused by a microdeletion $(1.5-3 \mathrm{Mb})$ of chromosome 22, with an incidence of 1 in 20004000 live births. The phenotype of this disorder is highly variable and common physical manifestations include craniofacial and cardiovascular anomalies, immunodeficiency, short stature and hypocalcaemia. In late adolescence and early adulthood, up to $50 \%$ of all individuals with 22q11.2DS develop schizophrenia or schizoaffective disorder. ${ }^{1}$ The 22q11.2 deletion is therefore one of the greatest known genetic risk factors for schizophrenia. However, penetrance is incomplete and the remaining 22q11.2DS carriers do not develop disease. We asked whether combinations of additional genomic flaws may be needed to develop schizophrenia and here we describe an individual with 22q11.2DS with schizophrenia, and in whom we detected two additional rare schizophrenia-associated genetic defects. The individual's written consent to publish was obtained.

\section{Method}

Previously, we screened 300 unrelated Japanese individuals with schizophrenia who met the DSM-III- ${ }^{2}$ diagnostic criteria for schizophrenia, by genotyping of markers D22S941, D22S944, D22S264 and D22S311. ${ }^{3}$ Concomitantly, we detected one patient with only one allele for each of these polymorphic markers. This individual was a female high-school graduate then aged 28 years. She was born by normal delivery without any obstetric complications. She was admitted to hospital at the age of 18 years for 2 months and again at the age of 25 years for 1 month. Her IQ, which was measured during her first hospital admission, was 61. We decided to re-evaluate her in August 2010. At this time, she was 37 years old and being treated with antipsychotics

*These two authors contributed equally to this work. (risperidone $5 \mathrm{mg} /$ day and quetiapine $95 \mathrm{mg} /$ day), but still suffered from persistent auditory hallucinations. Her only physical symptom was a slight hypocalcaemia $(8.4 \mathrm{mg} / \mathrm{dl}$ in serum, normal range $8.5-10.5 \mathrm{mg} / \mathrm{dl}$ ). She remains unmarried, lives in a group home and is employed in a semi-sheltered workplace. We re-examined her 22q11.2 microdeletion by fluorescence in situ hybridisation (FISH) with the TUBLE1 probe, and using the NimbleGen Human CNV 3x720K v1.0 Array (Roche Applied Science, Tokyo, Japan).

We recently reported that a subpopulation of individuals with schizophrenia have high plasma pentosidine levels (i.e. 'carbonyl stress') and in 2 such individuals out of 3682 participants, we detected frameshift mutations (P122LfsX27 and T27NfsX15) in the glyoxalase I (GLO1) gene, a zinc metalloenzyme, providing a possible explanation for the high plasma pentosidine levels. ${ }^{4}$ Therefore, we set out to measure pentosidine levels in our patient by high-performance liquid chromatography assay and perform a re-sequencing analysis of GLO1, as described previously. ${ }^{4}$

In another previous report, we showed an association between polyalanine stretch length polymorphisms of the paired-like homeobox $2 \mathrm{~b}$ (PMX2B or PHOX2B) gene encoding a transcription factor, with people who have schizophrenia, in particular those with strabismus. ${ }^{5}$ As our patient has constant exotropia in her right eye, we examined this gene: briefly, the genomic region encoding the 20-alanine tract was amplified using fluorescently labelled forward and reverse primers. Polymerase chain reaction products were run on an ABI 3130xl genetic analyser and the resulting data analysed using the Gene Mapper and Peak Scanner software (Applied Biosystems, Foster City, California, USA).

\section{Results}

The FISH data (online Fig. DS1(a)) and the microarray-based $\mathrm{CNV}$ analysis (not shown) revealed that the woman has a $3 \mathrm{Mb}$ hemizygous genomic deletion on chromosome 22q11.2, confirming that she has 22q11.2DS. An echocardiogram examination showed no abnormalities in the cardiovascular system (online Fig. DS2). We measured her plasma pentosidine levels twice, 1 week apart, and both values exceeded the mean +2 standard deviation levels of healthy controls $(n=61)^{4}$ (online Fig. DS1(b)). Then, we examined the GLO1 gene and identified a frameshift mutation 
(P122LfsX27; identical to one of our previously detected mutations $^{4}$ ) (online Fig. DS1(c)). An examination of the PHOX2B gene revealed a 5 -alanine deletion within the 20 -alanine stretch region (online Fig. DS1(d)).

\section{Discussion}

The frequencies of schizophrenia-associated CNVs are rare but generally have larger effects on susceptibility to the disease compared with risk-conferring single nucleotide polymorphisms. Among such CNVs, the 22q11.2 deletion has the deepest penetrance of 0.553 (95\% CI 0.18-0.97). ${ }^{6}$ The penetrance of other large effect size CNVs, for example the 15q13.3 deletion and 16 p11.2 duplication are 0.074 (95\% CI $0.03-0.16)$ and 0.069 (95\% CI $0.03-0.14)$ respectively. $^{6}$ Nonetheless, the $22 \mathrm{q} 11.2$ deletion is not sufficient to develop schizophrenia, and other risk factors are required. This case demonstrates for the first time to our knowledge that concrete additional risk variants could contribute to the manifestation of schizophrenia in combination with 22q11.2DS. In view of the penetrance of these mutations, this view has to be a qualified one as other causative mutations may contribute to this individual's developmental phenotypes.

We show that one contributing factor is the accumulation of pentosidine, an advanced glycation end-product resulting from carbonyl stress, a state featuring an increase in reactive carbonyl compounds and their attendant protein modifications. Our prior study demonstrated that people with schizophrenia have significantly higher plasma pentosidine levels than controls, and revealed that some participants with high pentosidine levels have impaired GLO1 activity, which is caused by a Glu111 $\rightarrow$ Ala amino acid change and frameshift mutations (see below). ${ }^{4}$ Cellular removal of reactive carbonyl compounds, including pentosidine, hinges largely on the activity of GLO1. ${ }^{7}$ The identified frameshift mutations were P122LfsX27 and T27NfsX15 (one each out of 1761 participants with schizophrenia), both of which elicit nonsense-mediated mRNA decay resulting in a reduction (to $40-50 \%)$ of GLO1 protein expression and activity. ${ }^{4}$ The current patient carries the P122LfsX27 frameshift mutation, coincidentally identical to one of our previously detected mutations, ${ }^{4}$ further confirming its relationship to disease.

The other genetic defect that we identified in this individual is a polyalanine length variation in the $P H O X 2 B$. We previously reported that constant exotropia displays a marked association with schizophrenia $(P=0.00000000906$, odds ratio $(\mathrm{OR})=20.6$, 95\% CI 4.83-87.8), and that deletion/insertion polymorphisms in the 20-alanine homopolymer stretch of $P H O X 2 B$ disturbs its transcription. ${ }^{5}$ We detected a modest association between these functional polymorphisms and all those with schizophrenia $(P=0.012, \quad$ OR $=1.59,95 \%$ CI $1.01-2.50)$ and those with schizophrenia and constant exotropia $(P=0.004, \mathrm{OR}=4.52,95 \%$ CI $1.78-11.5)^{5}$
In summary, we propose a combinatorial model for the genetic risk of schizophrenia where the combination of a $\mathrm{CNV}$, in this case 22q11.2DS, with a large effect; a GLO1 frameshift mutation with a probable moderate to large effect; and a 5-alanine deletion with a modest effect, collaborate to exceed a developmental threshold for the onset of schizophrenia. This proposal is the first discrete evidence for a multiple-hit hypothesis in schizophrenia, and although the current findings are fortuitous, the emerging availability of whole genome sequencing methodologies in the near future should help to systematically decipher the detailed genetic mechanisms that determine which individuals who are schizophrenia-associated CNV carriers go on to develop the disease, and to define its precise genetic spectrum.

M. Toyosima, PhD, M. Maekawa, MD, PhD, T Toyota, MD, PhD, Y, IWayama, MS, Laboratory for Molecular Psychiatry, RIKEN Brain Science Institute, Saitama; M. Arai, $\mathrm{PhD}$, T. Ichikawa, MD, M. Miyashita, PhD, Project for Schizophrenia Research, Tokyo Institute of Psychiatry, Tokyo; T. Arinami, MD, PhD, Department of Medical Genetics, Graduate School of Comprehensive Human Sciences, University of Tsukuba, Tsukuba, Ibaraki; M. Itokawa, MD, PhD, Laboratory for Molecular Psychiatry, RIKEN Brain Science Institute, Saitama, and Project for Schizophrenia Research, Tokyo Institute of Psychiatry, Tokyo; T. Yoshikawa, MD, PhD, Laboratory for Molecular Psychiatry, RIKEN Brain Science Institute, Saitama, Japan

Correspondence: Motoko Maekawa, Laboratory for Molecular Psychiatry RIKEN Brain Science Institute, 2-1 Hirosawa, Wako-city, Saitama 351-0198, Japan. Email: mmaekawa@brain.riken.jp

First received 3 Mar 2011, final revision 10 May 2011, accepted 16 Jun 2011

\section{Funding}

This work was supported by RIKEN BSI Funds, and a part of this study is the result of 'Development of biomarker candidates for social behavior' carried out under the Strategic Research Program for Brain Sciences by the MEXT of Japan.

\section{References}

1 Karayiorgou M, Simon TJ, Gogos JA. 22q11.2 microdeletions: linking DNA structural variation to brain dysfunction and schizophrenia. Nat Rev Neurosci 2010; 11: 402-16.

2 American Psychiatric Association. Diagnostic and Statistical Manual of Mental Disorders (3rd edn, revised) (DSM-III-R). APA, 1987.

3 Arinami T, Ohtsuki T, Takase $\mathrm{K}$, Shimizu H, Yoshikawa $\mathrm{T}$, Horigome $\mathrm{H}$, et al. Screening for 22q11 deletions in a schizophrenia population. Schizophr Res 2001; 52: 167-70.

4 Arai M, Yuzawa H, Nohara I, Ohnishi T, Obata N, Iwayama Y, et al. Enhanced carbonyl stress in a subpopulation of schizophrenia. Arch Gen Psychiatry 2010; 67: 589-97.

5 Toyota T, Yoshitsugu K, Ebihara M, Yamada K, Ohba H, Fukasawa M, et al. Association between schizophrenia with ocular misalignment and polyalanine length variation in PMX2B. Hum Mol Genet 2004; 13: 551-61.

6 Vassos E, Collier DA, Holden S, Patch C, Rujescu D, St Clair D, et al. Penetrance for copy number variants associated with schizophrenia. Hum Mol Genet 2010; 19: 3477-81.

7 Thornalley PJ. The glyoxalase system in health and disease. Mol Aspects Med 1993; 14: 287-371. 